\title{
Free Volume of PVA/SSA Proton Exchange Membrane Studied by Positron Annihilation Lifetime Spectroscopy
}

\author{
M.M. GomaA ${ }^{a, b, *}$, C. Hugenschmidt ${ }^{a}$, M. Dickmann ${ }^{a}$, M.O. Abdel-Hamed ${ }^{b}$, \\ E.E. ABDEL-HADY ${ }^{b}$ AND H.F.M. MOHAMED ${ }^{b}$ \\ ${ }^{a}$ Heinz Maier-Leibnitz Zentrum (MLZ) and Physik-Department E21, Technische Universität München, \\ Lichtenbergstraße 1, 85748 Garching, Germany \\ ${ }^{b}$ Physics Department, Faculty of Science, Minia University, P.O. Box 61519 Minia, Egypt
}

\begin{abstract}
Humidity control and water management in polymer electrolyte membranes for fuel cells are still of high importance to improve the fuel cells' efficiencies. In this study, poly (vinyl alcohol) (PVA)was crosslinked using 15 wt\% sulfosuccinic acid (SSA) by a solution casting method and additionally thermally crosslinked at $100{ }^{\circ} \mathrm{C}$. Positron annihilation lifetime spectroscopy was used to study the mean free volume size and the distribution at different humidity. A slight decrease in the free volume was found up to a relative humidity of $30 \%$ whereas it increases strongly for a relative humidity of more than $30 \%$. The volume of the voids duplicates from 0.036 to $0.078 \mathrm{~nm}^{3}$ by changing the relative humidity from 30 to $80 \%$. Thermogravimetric analyzer was used to determine the thermal stability of the membrane. From thermogravimetric analyzer data, it was found that the PVA with $15 \mathrm{wt} \%$ SSA membrane are chemically stable up to $200^{\circ} \mathrm{C}$.
\end{abstract}

DOI: 10.12693/APhysPolA.132.1519

PACS/topics: 82.47.Gh,78.70.Bj

\section{Introduction}

Fuel cell research and development is a very important topic in material science, since fuel cells are considered to be the power for a different application in the next years. The simple physical structure of a fuel cell consists of three main parts: cathode, anode, and electrolyte. Direct methanol fuel cells (DMFCs), which use methanol as a fuel, are used in many portable devices [1]. The electrolyte, also referred to as proton exchange membrane (PEM), should be a polymer material with stringent requirements: the membrane should exhibit high ion exchange capacity, high proton conductivity, and low methanol crossover. In addition, it should be stable at high mechanical load and at high temperature.

Crossover of methanol, hydrogen, or oxygen gas through the PEM leads to deterioration of the cell performance and degradation of the PEM [2] . Nafion ${ }^{\circledR}$ membrane is the most popular and commercial used PEM, firstly produced by DuPont in 1960 [3]. Beside its high proton conduction and high chemical stability, a serious drawback of Nafion ${ }^{\circledR}$ is the attribute that it is not stable above $100^{\circ} \mathrm{C}$. Moreover it shows a high methanol permeability (crossover) [4].

In general, the fuel permeability through PEM is believed to be correlated to the structure of the membrane and the open free volume in the PEM material [5-7], hence, the structure with less free volume is desirable for PEM. In order to develop advanced PEM materials with low fuel permeability, it is important to gain a deeper

*corresponding author; e-mail: mahmoud_gomaa19@mu.edu.eg insight into the nanostructure and, in particular, into the free volume hole size distribution. Positron annihilation lifetime spectroscopy (PALS) is a highly sensitive technique that allows, among other possibilities, to investigate the free open volume in the polymers and can, therefore, ideally be used to determine the pore sizes in PEMs. To investigate a positron lifetime spectrum, the time difference between positron implantation into a specimen and the annihilation is measured. After implantation, the positron is thermalized by inelastic scattering and diffuses afterwards freely through the material. During diffusion, the positron can undergo a number of processes, which lead sooner or later to the annihilation with an electron. One of these processes is the formation of a bound state with an electron called positronium (Ps). In polymers Ps is often formed due to the occurrence of pores. In this case, the corresponding lifetime spectrum includes three or more lifetime components $\tau_{1}, \tau_{2}$ and $\tau_{3}$ with relative intensities $I_{1}, I_{2}$ and $I_{3}$, respectively. The first two components are shorter lifetimes: $\tau_{1}$ is attributed to the para-positronium $(p-\mathrm{Ps})$ with a vacuum lifetime of $125 \mathrm{ps}, \tau_{2}$ is the annihilation of a free positron inside the material with a lifetime of about $450 \mathrm{ps}$. The third component is the longest lifetime, it is due to ortho-positronium (o-Ps) annihilation with a lifetime of more than 1 ns. $\tau_{3}$ is strongly correlated with the size of the pores where it annihilates via a pick-off reaction [8]. Therefore, the information of free volume structures in the materials can be obtained by PALS. Assuming spherical pore shapes, the radius can be simply calculated using a semi-empirical relation from the Tao-Eldrup model [9, 10]: 


$$
\tau_{3}=\frac{0.5}{1-R / R_{0}+\sin \left(2 \pi R / R_{0}\right) /(2 \pi)} \mathrm{ns},
$$

where $R_{0}=R+\Delta R$ is the radius of an infinitely deep square well potential and $\Delta R=0.166 \mathrm{~nm}$ is the thickness of the homogeneous electron layer in which the $o$-Ps annihilates by conversion into $p$-Ps [11]. The hole volume is obtained using the equation

$$
V_{h}=4 \pi R^{3} / 3 \text {. }
$$

This model is successfully applied to investigate the free volume of many polymer materials [12]. A lot of experiments have been carried out to study the diffusion of gas and methanol molecules through the polymer matrix [13-15].

In the present work, the effect of humidity on the size of the free volume of the poly (vinyl alcohol) (PVA) with $15 \%$ sulfosuccinic acid (SSA) membrane has been studied using PALS.

\section{Experimental \\ 2.1. Membrane preparation}

PVA [Alfa Aesar] 98-99\% hydrolyzed and high molecular weight $\left(M_{w}: 89000\right)$, sulfosuccinic acid (SSA) [70 wt\% solution, Aldrich] were used without purification. The crosslinked PVA/SSA proton exchange membrane was prepared using casting method [16]. PVA (10 wt\%) was dissolved in deionized water under stirring at $80^{\circ} \mathrm{C}$ for $6 \mathrm{~h} .15 \mathrm{wt} \% \mathrm{SSA}$ was added to the PVA homogeneous solution. To be sure that the solution was fully dissolved, PVA/SSA solution was kept under stirring for $24 \mathrm{~h}$ at room temperature $\left(25^{\circ} \mathrm{C}\right)$. The solution was then poured onto Teflon Petri dish which is used to avoid sticking to the surface for easier removing and then the excess water solvent was evaporated in a vacuum oven at $60^{\circ} \mathrm{C}$. After evaporation, the PVA/SSA polymer membranes were crosslinked thermally at $100{ }^{\circ} \mathrm{C}$ for $1 \mathrm{~h}$ and stored in bags for further measurements.

\subsection{Thermal analyses}

A thermogravimetric analyzer (TGA) instrument model Q50-USA was used to characterize the thermal properties of the membranes. Measurements were performed by heating the sample under a nitrogen atmosphere from 35 to $700^{\circ} \mathrm{C}$ with a heating rate of $10^{\circ} \mathrm{C} / \mathrm{min}$.

\subsection{Positron lifetime measurements}

Positron annihilation lifetime measurements are carried out using ${ }^{22} \mathrm{NaCl}$ radioactive material enveloped between $25 \mu \mathrm{m}$ Kapton foil. The source activity is about $0.297 \mathrm{MBq}$. The source is sandwiched between 10 layers of the membrane with a total thickness $1 \mathrm{~mm}$ on each side to ensure that most of the positrons completely annihilate inside the membrane material. Using a fastfast coincidence spectrometer with a time resolution of 340 ps, spectra were collected containing more than 3 million counts each. PALSfit3 was used for analyzing these spectra [17]. The source correction contribution was carried out using a well-annealed aluminum specimen with $99.00 \%$ purity and it was found to be $17.9 \%$.

\section{Results and discussion}

\section{1. $T G A$}

Figure 1 shows the TGA thermograph of PVA and $15 \mathrm{wt} \%$ of SSA with additional thermally crosslinked at $100{ }^{\circ} \mathrm{C}$. The TGA curve of PVA and $15 \mathrm{wt} \%$ of SSA shows three main regions, where the first part, attributed to weight loss of absorbed water, is located between 50 and $210^{\circ} \mathrm{C}$. The second loss region between 210 and $420^{\circ} \mathrm{C}$ arises from the breakage of sulfuric acid group and the ester bonds. The last region between 420 and $500^{\circ} \mathrm{C}$ issues from the oxidation process in the polymer. From TGA curve, the PVA with 15 wt $\%$ SSA are chemically stable up to $200^{\circ} \mathrm{C}$ which is suitable for proton exchange membrane fuel cell.

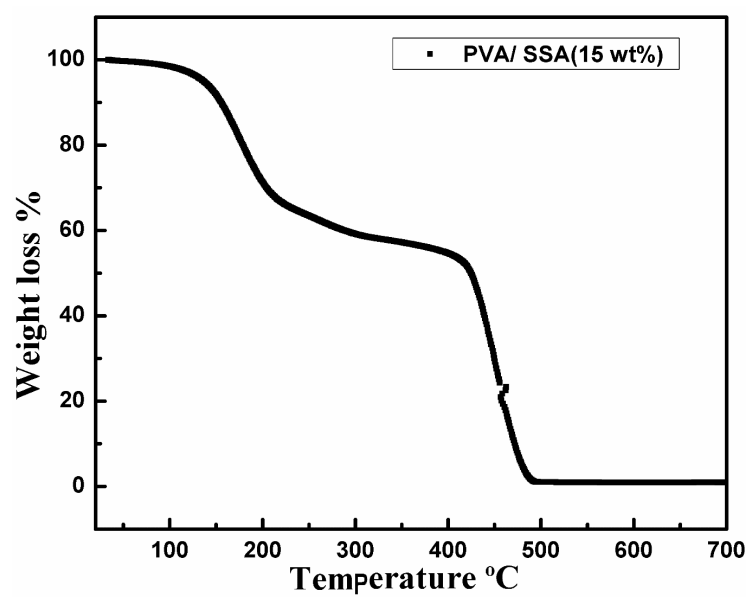

Fig. 1. TGA curve of PVA with 15 wt\% SSA.

\subsection{Positron lifetime measurements}

PVA/SSA (15 wt\%) was dried under vacuum for one day and then humidified at different humidity for $24 \mathrm{~h}$ before starting the experiment in order to reach equilibrium conditions. Figure 2 shows the effects of the humidity from zero to $80 \%$ relative humidity $(\mathrm{RH})$ on the positron lifetime in PVA with 15 wt\% SSA concentrations. The positron lifetime data are transformed into mean hole size values using Eq. (1). It is observed that at low humidity between 0 to $30 \% \mathrm{RH}$, there is a slight decline in the $o$ Ps lifetime and corresponding decrease in the mean free volume size. However, at higher humidity (30-80\% RH), the $o$-Ps lifetime increases significantly and the size of the free volume is duplicated from 0.036 to $0.078 \mathrm{~nm}^{3}$ when humidity changed from 30 to $80 \% \mathrm{RH}$. It was observed that there is an agreement between this behavior and the previous study of humidity effects on Polyamide 6 (PA6) $[18,19]$. At the low humidity region, with increase of humidity up to $30 \% \mathrm{RH}$, water molecules start filling intermolecular spaces in the membrane, which is seen as the reason for a slight decrease of the free volume. On the other hand, at a humidity of more than $30 \% \mathrm{RH}$, the plasticizing effect of water molecules is responsible for the increase of free volume sizes. 


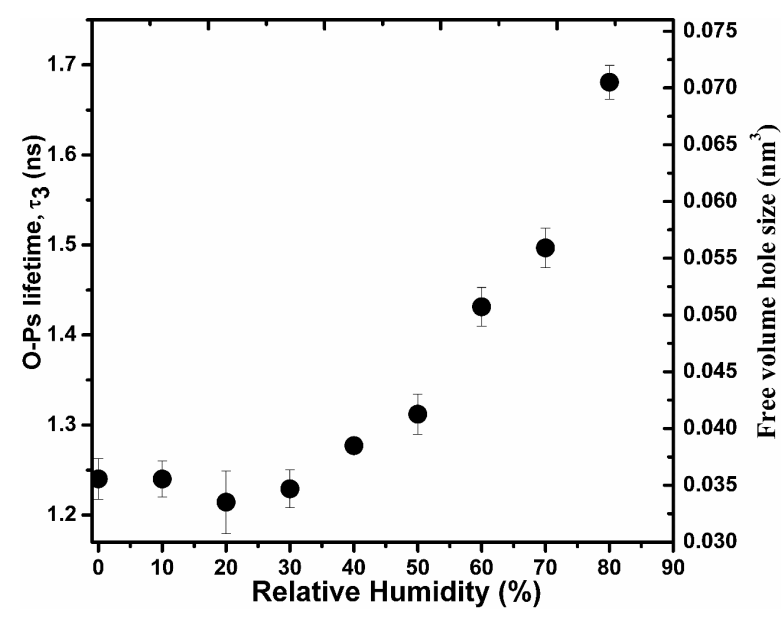

Fig. 2. The $o$-Ps lifetime $\tau_{3}$ and mean void size at a different humidity of PVA/SSA (15 wt\%) membrane.

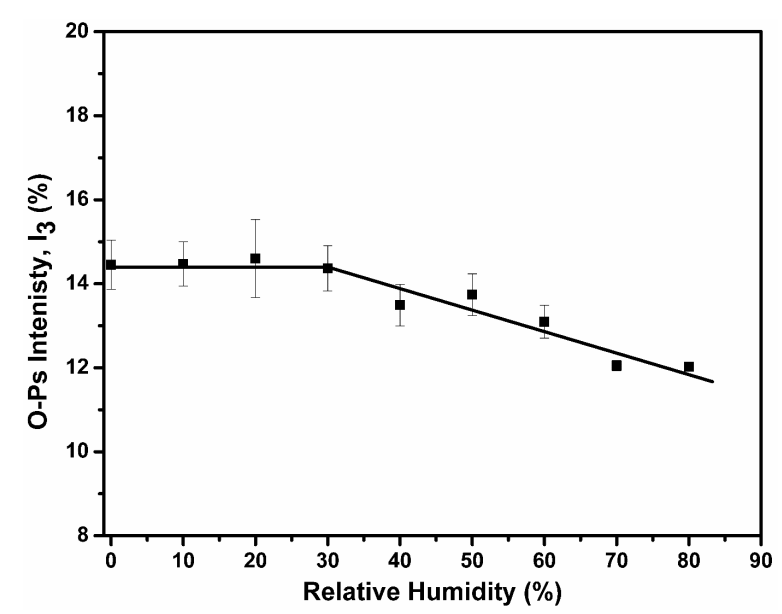

Fig. 3. The $o$-Ps intensity $I_{3}$ at a different humidity of PVA/SSA (15 wt\%) membrane.

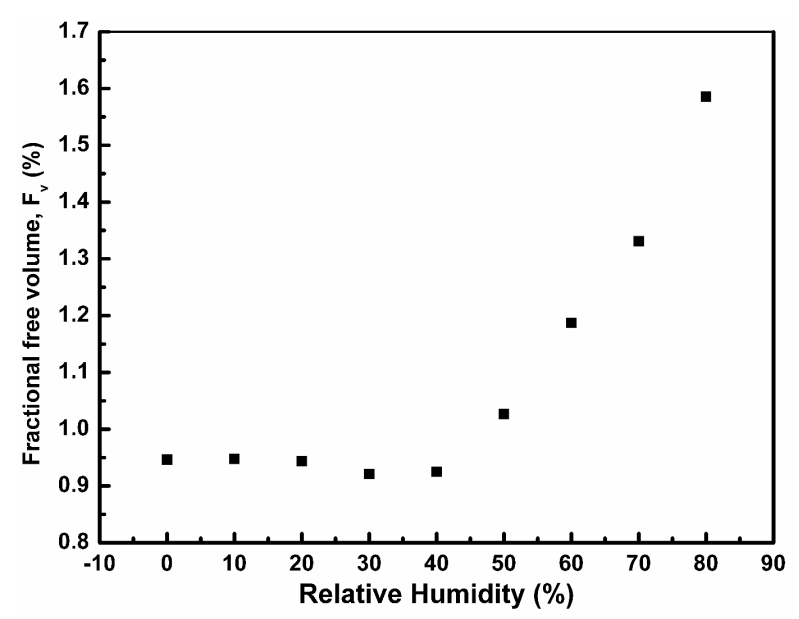

Fig. 4. Fractional free volume $F_{v}$ for PVA/SSA (15 wt\%) membrane at different humidity.

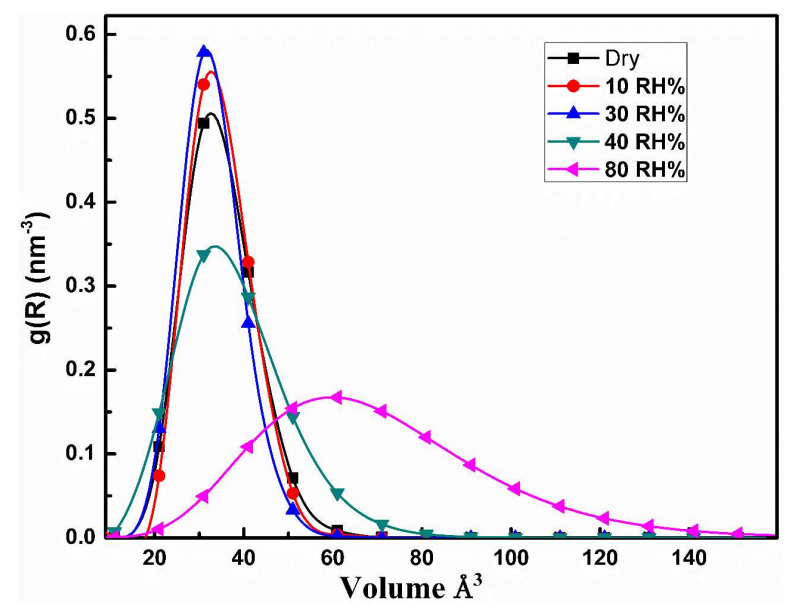

Fig. 5. Probability density function $g(R)$ vs. volume of free volume holes for PVA/SSA (15 wt\%) membrane at different humidity.

Figure 3 shows the $o$-Ps intensity $I_{3}$ as a function of relative humidity at room temperature $\left(25^{\circ} \mathrm{C}\right)$. It is evident from the figure that there are no changes in the $o$-Ps intensity at low humidity and $I_{3}$ started decreasing after $30 \% \mathrm{RH}$. From $I_{3}$ behavior, at low humidity (below $30 \% \mathrm{RH}$ ), no significant changes were observed in the $o$-Ps formation. On the other hand, at high humidity region, the free volumes recombine together and Ps may be trapped into water molecules in addition to the material itself.

The fractional free volume $F_{v}$ is the ratio between the free volume to the total volume of the material and can be calculated using the equation

$$
F_{v}=C V_{h} I_{3}
$$

where $C$ is a constant equal to $0.018 \mathrm{~nm}^{-3}, V_{h}$ is the mean free volume of the voids and $I_{3}(\%)$ is the o-Ps intensity.

The fractional free volume decreases from zero up to $30 \% \mathrm{RH}$, then increases at high humidity (more than $30 \% \mathrm{RH}$ ) as shown in Fig. 4. At low humidity or, in other words, at a low water uptake, the free water molecules occupy the spaces in the membrane which appeared on decrease of the fractional free volume. At high humidity, high water uptake is responsible for the significant increase in the fractional free volume, because water molecules work as spacer agent between polymer chains.

The advantage of the continuous lifetime analysis is that one can obtain free volume hole size distribution, rather than the average values obtained by finite analysis. PALSfit3 was used for the calculation of the free volume distribution. Figure 5 shows this distribution for PVA/SSA (15 wt\%) at room temperature $\left(25^{\circ} \mathrm{C}\right)$ and at different relative humidity up to $80 \%$ relative humidity. The free volume distribution was determined by analyzing the $o$-Ps lifetime from zero humidity up to $80 \% \mathrm{RH}$. At low RH up to $30 \%$ the void size distribution becomes more narrow. The hole volume distribution is between 10 and $87 \AA^{3}$ for the dry membrane and between 12 and 
$61 \AA^{3}$ at $30 \% \mathrm{RH}$, where, in addition, the area under the peak decreases indicating a reduction of the free volume fraction. The peak slightly shifts to the left side (to a lower value), meaning a small decrease of the mean void size. Above $30 \% \mathrm{RH}$, the area under the peak increases and the spectrum becomes broader and there are significant changes in the hole volume distribution, which become between 10 and $157 \AA^{3}$ for $80 \% \mathrm{RH}$. This reflects an increase in the free volume fraction and also the peak shifted to a higher value, meaning an increase in the average free volume holes size.

\section{Conclusion}

PALS was used to determine the mean size and the distribution of voids at different humidity for PVA/SSA (15 wt\%). Two regions of changing the free volume with the humidity were found and attributed to different processes. In the first region at low humidity below $30 \% \mathrm{RH}$ the mean void size slightly decreases due to water filling of preexisting holes in the membrane. At more than $30 \% \mathrm{RH}$ the significant increase in the free volume is attributed to the plasticizing effect of water molecules in PVA/SSA (15 wt\%). TGA results revealed that PVA with 15 wt $\%$ SSA are chemically stable up to $200^{\circ} \mathrm{C}$ which is suitable for proton exchange membrane fuel cell.

\section{Acknowledgments}

The first author would like to thank Egyptian Ministry of High Education for providing the financial support (Ph.D. scholarship) for this research.

\section{References}

[1] T. Zhang, Q.-M. Wang, J. Power Sources 140, 72 (2005).
[2] A. El-Kharouf, T.J. Mason, D.J.L. Brett, B.G. Pollet, J. Power Sources 218, 393 (2012).

[3] O. Savadogo, J. New Mater. Electrochem. Syst. 1 , 47 (1998).

[4] A. Kraytsberg, Y. Ein-Eli, Energy Fuels 28, 7303 (2014).

[5] C. Nagel, K. Günther-Schade, D. Fritsch, T. Strunskus, F. Faupel, Macromolecules 35, 2071 (2002).

[6] Y. Kobayashi, K. Haraya, S. Hattori, T. Sasuga, Polymer 35, 925 (1994).

[7] H.F.M. Mohamed, K. Ito, Y. Kobayashi, N. Takimoto, Y. Takeoka, A. Ohira, Polymer 49, 3091 (2008).

[8] P. Winberg, K. DeSitter, C. Dotremont, S. Mullens, I.F.J. Vankelecom, F.H.J. Maurer, Macromolecules 38, 3776 (2005).

[9] M. Eldrup, D. Lightbody, J.N. Sherwood, Chem. Phys. 63, 51 (1981).

[10] S.J. Tao, J. Chem. Phys. 56, 5499 (1972).

[11] H. Nakanishi, in: Int. Symp. on Positron Annihilation Studies of Fluids, Ed.: S.C. Sharma, World Scientific, 1988, p. 292.

[12] Y. Kobayashi, Def. Diff. Forum 331, 253 (2012).

[13] E. Abdel-Hady, M. Abdel-Hamed, M. Eltoony, A. Hammam, M. Elsharkawy, J. Phys. Conf. Series 262, conf. 1 (2011).

[14] S.J. Osborn, M.K. Hassan, G.M. Divoux, D.W. Rhoades, K.A. Mauritz, R.B. Moore, Macromolecules 40, 3886 (2007).

[15] Y. Ito, Radiat. Phys. Chem. 64, 375 (2002).

[16] S.L. Chen, L. Krishnan, S. Srinivasan, J. Benziger, A.B. Bocarsly, J. Membr. Sci. 243, 327 (2004).

[17] P. Kirkegaard, J.V. Olsen, M.M. Eldrup, PALSfit3: A software package for analysing positron lifetime spectra, Kgs. Lyngby: Technical University of Denmark (DTU), Ris $\emptyset 2017$.

[18] G. Dlubek, F. Redmann, R. Krause-Rehberg, J. Appl. Polym. Sci. 84, 244 (2002).

[19] W. Zhou, Z. Chen, N. Oshima, K. Ito, B.E. O'Rourke, R. Kuroda, R. Suzuki, H. Yanagishita, T. Tsutsui, A. Uedono, Appl. Phys. Lett. 101, 014102 (2012). 